\title{
Molecular Docking Simulation of Short-Chain Four Disulphide Bridged Scorpion Toxins with Structural Model of Human Voltage-Gated Potassium Ion Channel Kv1.3
}

\author{
Sudandiradoss C. and Rao Sethumadhavan* \\ Bioinformatics Division, School of Biotechnology, Chemical and Biomedical Engineering, Vellore Institute of \\ Technology University, Vellore 632014, Tamil Nadu, India
}

\begin{abstract}
We report structural model of the human voltage-gated potassium ion channel Kv1.3 obtained based on the crystallographic structure of KcsA by homology modeling. Molecular docking simulations were performed between the model structure of Kv1.3 channel with three short-chain four disulphide bridged scorpion toxins HsTX1 from the venom of Heterometrus spinnifer (Scorpionidae), maurotoxin (MTX) from Scorpiomaurus palmatus and Pandinus toxin 1(Pi1) from Pandinus imperator which belongs to the $\alpha$-KTx6 subfamily. By integrating the homology modeling and docking simulations we obtained the three dimensional structures of toxin-channel complexes. The final docked complexes were then subject to minimization with CHARMM force field and investigated key interacting residues, electrostatic interaction energies, binding free energies, disulphide bridge pairing determination, folding pattern, hydrogen bond formation, hydrophobic contacts and flexibilities between selected scorpion toxins to the Kv1.3 channel.
\end{abstract}

Keywords: $\mathrm{K}^{+}$channel, homology modeling, scorpion toxins.

\section{INTRODUCTION}

Ion channels are found in organisms ranging from viruses, bacteria to mammals and play a key role in the transduction of electric signals [1], control of ionic concentrations and membrane potentials. Potassium channels are the most diverse group of ion channel family [2] and play fundamental roles in physiology by controlling the electrical activity of excitable cells [1]. Different approaches are devoted to the structural and functional characterization of $\mathrm{K}^{+}$channels. The best characterized $\mathrm{K}^{+}$channels are derived from homolog of the Drosophila $\mathrm{K}^{+}$channel genes including shaker, shab, shaw, and shal which exist in mammalian tissues and were assigned as $\mathrm{Kv} 1, \mathrm{Kv} 2, \mathrm{Kv} 3$, and $\mathrm{Kv} 4$ respectively [3-6]. Each type of $\mathrm{Kv}$ channels may be divided into different subtypes [1]. For the Kv1 channels, the voltage-gated potassium channel, Kv1.3, plays an important role in T-cell physiology because of its involvement in the regulation of membrane potential of cells and influencing Tcell activation in vitro [7-10]. The Kv1.3 channel is one of two potassium channels expressed by human $\mathrm{T}$ lymphocytes that are involved in proliferation and cytokine secretion, the other is the calcium-activated $\mathrm{K}^{+}$channel IKCa1 .

In the management of autoimmune diseases and in the prevention of graft rejection following organ transplants, immunosuppressants are routinely used. Clinically useful immunosuppressants might be developed by selectively targeting lymphocyte potassium channels [11-13]. Immunosuppression through highly selective blockade of the

\footnotetext{
*Address correspondence to this author at the Bioinformatics Division, School of Biotechnology, Chemical and Biomedical Engineering, Vellore Institute of Technology University, Vellore 632014, Tamil Nadu, India; Tel: +91 4162202522; Fax: +91 4162243092;

E-mail: rsethumadhavan@vit.ac.in
}

T-cell potassium channels is attractive because of three reasons (i) due to the functionally restricted tissue distribution of Kv1.3, (ii) an important role in T-cell activation and (iii) the availability of specific and potent inhibitors of the channel. The human Kv1.3 has been suggested to be an excellent target for modulating immune system functions by specific and potent blockers in the therapeutic management of stroke, epilepsy and cardiac arrhythmias $[7,8,10,14]$. Therefore, it is significant to study the action of toxins on Kv1 channels.

The scorpion toxins have been useful molecules in modulating the potassium channel structures and functions [15-20]. A number of scorpion $\mathrm{K}^{+}$channel inhibitors have been previously characterized that target the shaker-related subfamily of voltage-gated $\mathrm{K}^{+}$channels and the $\mathrm{Ca}^{2+}$ dependent $\mathrm{K}^{+}$channels [21-24]. Those toxins are composed of a single polypeptide chain of 31-39 amino acid residues, reticulated by three conserved disulphide bridges $[25,26]$. To date, a variety of experimental strategies have defined functional domains within the Kv1 channels and thermodynamic mutant analyses have been used to identify the specific residues in the S5-S6 linker region, which is a part of the scorpion-toxin receptor site. The homotetrameric arrangement of voltage-gated potassium channels was first revealed by the blocking analyses of charybdotoxin (ChTX, from Leiurus quinquestriatus) and many other potent polypeptide inhibitors of the lymphocyte potassium channels have been discovered in scorpion venom [27-31]. ChTX was the first polypeptide shown to block all $\mathrm{Kv}$ channels, inhibiting Kv1.3 with nanomolar affinity. Other scorpion toxins include Noxiustoxin, Kaliotoxin, Margotoxin, Agitoxin-2, Hongotoxin, HsTX1, Maurotoxin, and Pandinus toxins 1-3 Pi1, Pi2, and Pi3 [29, 31-34]. Generally, channel inhibitors can be pore blockers or gating modifiers [21, 31 , 
35]. Pore blockers bind to the channel in $1: 1$ stoichiometry and plug the pore of the channel impeding the flow of the ionic current. These toxins are small proteins that block the passage of $\mathrm{K}^{+}$ions by binding at the pore entryway on the extracellular side of the channel, thereby inhibiting the ion flux. Gating modifiers such as hanatoxin (HaTx) block the $\mathrm{K}^{+}$current by modifying the gating mechanism of the channel. HaTx shifts the channel opening to more depolarized membrane potentials. One HaTx molecule can bind to each of the four subunits of the channel. The interactions of toxins with potassium channels are among the strongest and most specific known protein-protein complexes [17].

Many questions are still unanswered due to experimental difficulties and the lack of significant theoretical guidance. The majority of drugs marketed that act on ion channels were discovered empirically rather than by molecular insight, and most of them have shown serious problems of safety and efficacy $[36,37]$. Computational simulation at the molecular level can be a powerful tool in understanding electrophysiological experiments performed on potassium channels. However, no experimental data for the structure of scorpion toxins-Kv1.3 channel complexes have been reported.

In this study, we have integrated homology modeling, docking analysis and molecular dynamics simulations to understand the association of four-disulphide bridged scorpion toxins to the Kv1.3 channel. First, we have constructed the three-dimensional (3D) structure model for the Kv1.3 potassium channel via homology modeling, taking the $\mathrm{x}$-ray crystal structure of the KcsA potassium channel as a template. Then the docking analysis and molecular dynamics simulations were employed to localize the regions of binding and to identify the residues involved in the complex formation and to estimate the binding affinity of the Kv1.3 channel with three scorpion toxins, namely, a new toxin, HsTX1 from the venom of Heterometrus spinnifer (Scorpionidae), Maurotoxin (MTX) from Scorpiomaurus palmatus and Pandinus toxin 1(Pi1) from Pandinus imperator which belongs to the $\alpha$-KTx 6 subfamily [38].

\section{MATERIALS AND METHODS}

\section{Atomic Coordinates}

The atomic coordinates of the three scorpion toxins HsTX1 [39], MTX [40], and Pi1 [41] were obtained from the Brookhaven Protein Data Base [42] and their PDB entries are $1 \mathrm{QUZ}, 1 \mathrm{TXM}$ and $1 \mathrm{WZ} 5$ respectively.

\section{Model Protein}

Since the crystal structure of the human potassium channel Kv1.3 has not been determined, the threedimensional model of the Kv1.3 channel was obtained by using the homology modeling based on the KcsA crystal structure (PDB ID:1BL8) as template. The sequence alignment of KcsA (1BL8) with the Kv1.3 channel generated by CLUSTAL $\mathrm{W}$ [43] shows that the sequence identity between the two channels is $30.93 \%$, and the similarity is $62.89 \%$. The amino acid sequences of these two proteins are very similar especially their sequences in the pore region and extracellular entryway and thus the x-ray structure of the KcsA channel was used as a template for constructing the 3D structure model of the Kv1.3. The 3D structure model of the Kv1.3 channel was generated by the biopolymer homology modeling software of Swiss-model/Deep view 3.7. based on the KcsA structure. The sequence alignment of Kv1.3 with the KcsA (1BL8) was generated by CLUSTAL W [43].

\section{Molecular Docking Simulations}

Molecular docking simulations were performed using the protein-protein docking software GRAMM v 1.03 [44]. The algorithm used by GRAMM v 1.03 performs Fast Fourier transformation methodology by employing smoothed potentials, refinement stage and knowledge based scoring. The CHARMM 34 force field [45] was also used to assign the charges of the Kv1.3 channel and to the scorpion toxins. We used a fine-grid projection of a softened Lennard-Jones potential function [46] and then the top 4000 grid-based predictions are subjected to a conjugate minimization in continuous $6 \mathrm{D}$ rigid body space with the same soft potential. One representative prediction for each minimum is stored and the number of initial predictions falling into this minimum is marked as the volume of the minimum. The average radius of such minima on our smoothed landscape is $5 \AA$. The 15 best docked complexes were selected according to the global score from GRAMM v 1.03 and the global orientation relative to electrostatic properties of both toxins and channel. Swiss PDB viewer was used to determine electrostatic properties using coulomb computational method. The final docked complexes were minimized with a CHARMM 34 force field to relieve possible steric clashes and overlaps. During structural refinement, a distancedependent dielectric constant of 4 was used.

\section{Computation of Binding Free Energy}

The binding free energy of a tetramer Kv1.3 channel and scorpion toxin is obtained as follows:

$$
\Delta \mathrm{G}_{\text {bind }}=\mathrm{G}_{\text {complex }}-\left(\mathrm{G}_{\text {channel }}+\mathrm{G}_{\text {toxin }}\right)
$$

Since the residues at the interface contribute most to $\Delta \mathrm{G}_{\text {bind }}$ the total atom-atom potential of mean force, $G$, for each structure is calculated using the following formula

$$
\Delta G_{\text {bind }}=\frac{1}{2} \sum_{i j}^{\text {interface }} \bar{u}\left(i, j, r_{i j}\right)
$$

where the summation is over atomic pairs that are not in the same residue and a factor of $1 / 2$ is used to avoid doublecounting of residue-residue and atom-atom interactions.

We used DFIRE [47] software for calculating binding affinity provided by the State University of New York at Buffalo, New York.

\section{Computation of Disulphide Bridge Pairing Determination and Folding Pattern in Scorpion Toxins}

Disulphide bridges play a major role in the stabilization of the folding process and, consequently, in studies related to structural and functional properties of specific proteins. In addition, knowledge about the disulphide bonding state of cysteines may help the experimental structure determination 
process and may be useful in other genomic annotation tasks. We have used Ferre and Clote [48] method which follows feed forward neural-network architecture with hidden units associated with cysteine pairs and inputs encoding secondary structure. We used DiANNA [49] server for computing the disulphide bridge pairing determination.

Scorpion toxins are structured by a high number of disulphide bridges (from 2 -5) as compared to their backbone length, thereby conferring some rigidity to the molecules, a stabilization of their secondary structures, as well as a relative resistance to denaturation. Despite this peptide rigidity, there are some flexible domains more or less conserved could be diverted from their regular molecular roles to the activity such as ion channel recognition. Therefore, a better grasp on toxin folds offer valuable perspectives in the use of toxins as ion channel blockers. We used PFP-Pred [50] to locate the folding patterns in the selected scorpion toxins.

\section{Computation of Hydrogen Bond and Hydrophobic Contacts}

When two atoms bearing partial negative charges share partially positively charged hydrogen, the atoms are engaged in a hydrogen bond (H-bond). The correct 3-D structure of a protein is often dependent on an intricate network of $\mathrm{H}$ bonds. Similarly the hydrophobic contacts between toxins and ion channel complexes are also very essential. So, we computed the amino acid residues involved in hydrophobic contacts and hydrogen bond formation between the scorpion toxins and Kv1.3 channel. We used imoltalk [51] program for this purpose.

\section{Computation of Flexibilities of Scorpion Toxins with Kv1.3 Channel}

Normal mode analysis (NMA) is a powerful tool for predicting the possible flexibilities of a given macromolecule. Applications of NMA cover wide areas of structural biology, such as the study of protein conformational changes upon ligand binding, membrane channel opening and closure, potential movements of the ribosome, and viral capsid maturation. We have used ElNe'mo [52], a web interface of Elastic Network Model to measure the flexibilities of the scorpion toxins upon binding to the Kv1.3 channel during docking simulations.

\section{RESULTS}

\section{Homology Modeling of Human Voltage-Gated Potassium ion Channel Kv1.3}

We obtained the three-dimensional model of the Kv1.3 channel by using the homology modeling software of Swissmodel/Deep view 3.7 based on the KcsA crystal structure (PDB ID: 1BL8) as template. The source organism for KcsA crystal structure is Streptomyces lividans. The KcsA crystal structure has 160 amino acid residues with the molecular weight of $17.7 \mathrm{KDa}$. The Kv1.3 channel has 523 amino acid residues with the molecular weight of $58.3 \mathrm{KDa}$. The detail information for four subunits of KcsA and Kv1.3 are

Table 1. The Detailed Information of the KesA and Kv1.3 Channels

\begin{tabular}{|c|c|c|c|c|c|c|}
\hline K+ Channel & No. of a.a & Molecular Weight (KDa) & Gene Name & Source Organism & Swiss-Prot Accession Number & PDB ID \\
\hline $\mathrm{Kcs} A$ & 160 & 17.7 & $\mathrm{KCSA}$ & Streptomyces lividans & Q54397 & 1BL8 \\
\hline $\mathrm{Kv} 1.3$ & 523 & 58.3 & KCNA3 & Homo sapiens & P22001 & - \\
\hline
\end{tabular}

(a)

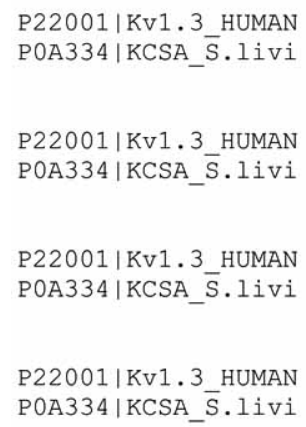

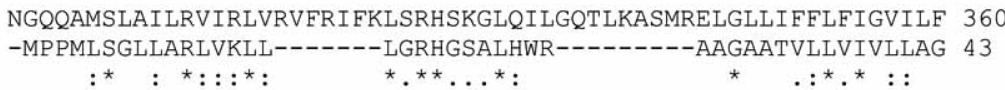

(b)

MTX -VSCTGSKDCYAPCRKQTGCPNAKCINKSCKCYGC-NH 34

Pi1 LVKCRGTSDCGRPCQQQTGAPNSKCINRMCKCYGA-NH 35

HsTX1-ASCRTPKDCADPCRKETGCPYGKCMNRKCKCNRC-NH 34

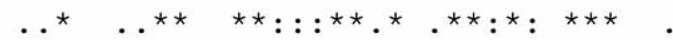

Fig. (1). Sequence alignment of (a) KcsA (1BL8) with Kv1.3 channel and (b) scorpion toxins generated by ClustalW. In the sequences, an asterisk indicates an identical or conserved residue, a colon indicates a conserved substitution, and a period indicates a semi conserved substitution. 


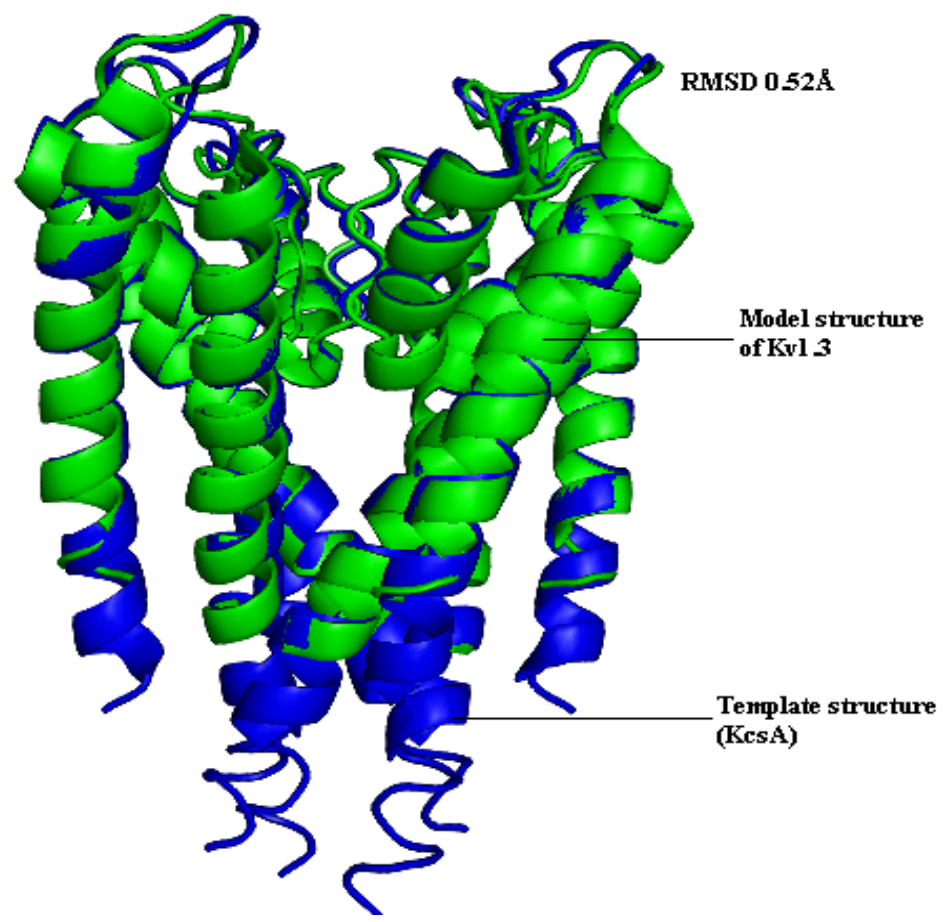

Fig. (2). The superimposed structures of KcsA crystal structure (PDB ID:1BL8) as template and three-dimensional model of the Kv1.3 channel with RMSD of $0.52 \AA$.

depicted in Table 1. The sequence alignment of KcsA (1BL8) with the Kv1.3 channel generated by CLUSTAL W [43] shows that the sequence identity between the two channels is $30.93 \%$, and the similarity is $62.89 \%$. The superimposition between KcsA (1BL8) with the model structure of Kv1.3 channel showed RMSD of $0.52 \AA$. The sequence alignment between KcsA (1BL8) with Kv1.3 channel and among the scorpion toxins are shown in Fig. (1a) and (1b) respectively. The superimposed structures of KcsA with the three-dimensional model of Kv1.3 channel with RMSD are shown in Fig. (2).
Molecular Docking of Four-Disulphide Bridged Scorpion Toxins to the Voltage-Gated Potassium ion Channel Kv1.3

We performed a multiple contact analysis to obtain favorable Kv1.3-toxin complexes for each structure of the scorpion toxins interacting with the Kv1.3 channel. The predicted HsTX1-Kv1.3, MTX-Kv1.3 and Pi1-Kv1.3 complexes are shown in Fig. (3). The most favorable contact in all complexes satisfying the criteria that the association distances between two proteins is less than $3 \AA$. We then analyzed the pairs of amino acids between the scorpion

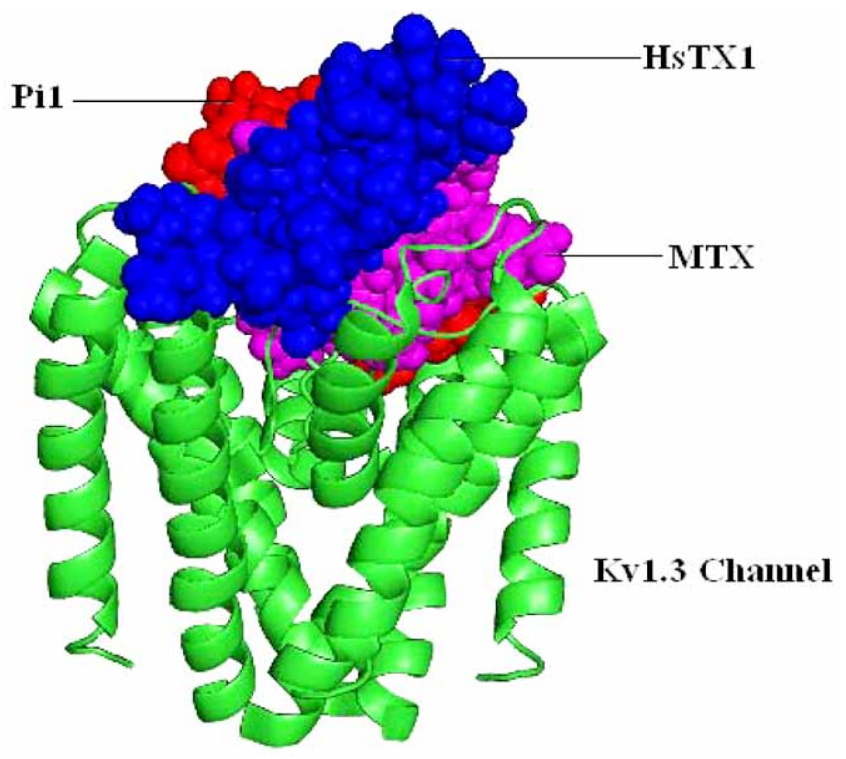

Fig. (3). Docked complexes of three-dimensional model of the Kv1.3 channel with HSTX1(blue), MTX(Magenta) and Pi1(red) scorpion toxins. 
toxins and the Kv1.3 channel using a new contact distance 5 $\AA$. The most frequent residues of the four disulphide bridged scorpion toxins occurred during the binding to the Kv1.3 channel are depicted in the Table 2. Among the three selected scorpion toxins the HSTX1 has the maximum number of 9 residues which have the contacts with Kv1.3 channel and the remaining two toxins namely MTX and Pi1 has 7 residue contacts. The electrostatic interaction energies between the scorpion toxins and Kv1.3 channel were calculated using the following process. The charges of the toxins were assigned onto the electrostatic potential grids of the Kv1.3 channel, and then the electrostatic interaction energies were calculated. The calculation results are listed in Table 3. From this table, it could be seen that, the electrostatic potential energy is high in HSTX1 and low in Pi1.

Table 2. Most Frequent Residues of the Four Disulfide Bridged Scorpion Toxins Occurred during the Binding to the Kv1.3 Channel

\begin{tabular}{|c|c|}
\hline Toxin & Residues \\
\hline \hline HsTX1 & Arg-4, Ly-7, Asp-8, Arg-14, Lys-15, Lys-23, Arg-27, Lys- \\
& 28, Lys-30 \\
\hline MTX & Thr-4, Lys-7, Arg-14, Lys-15, Lys-23, Lys-27, Lys-30 \\
\hline Pi1 & Lys-3, Arg-5, Thr-7, Arg-12, Lys-24, Arg-28, Lys-31 \\
\hline
\end{tabular}

Table 3. Electrostatic Properties of Contact Residues of both the Toxin and Channel Amino Acid Residues

\begin{tabular}{|c|c|c|}
\hline Toxins & Kv1.3 Channel-Toxin Residues & $\mathbf{E}_{\text {elec }}$ \\
\hline \hline HsTX1 & Asp-398-Lys-7 & -10.45 \\
\hline & Asp-398-Lys-15 & -10.23 \\
\hline & Asp-370-Arg-4 & -10.07 \\
\hline & Asp-370-Arg-14 & -9.18 \\
\hline MTX & Asp-398-Lys-28 & -10.08 \\
\hline & Asp-398-Thr-4 & -9.54 \\
\hline & Asp-398-Arg-14 & -9.72 \\
\hline & Asp-398-Lys-15 & -9.62 \\
\hline Pi1 & Asp-370-Lys-27 & -9.81 \\
\hline & Asp-398-Lys-3 & -8.83 \\
\hline & Asp-370-Arg-12 & -8.72 \\
\hline & Asp-398-Arg-28 & -8.90 \\
\hline & Asp-398-Lys-31 & -8.76 \\
\hline
\end{tabular}

Eelec -the electrostatic interaction energy between $\mathrm{Kv1} .3$ and the toxins (kcal/mol)

\section{Binding Free Energy Calculations}

The binding affinity of a Kv1.3 channel with scorpion toxin tetramer is obtained as follows:

$$
\Delta \mathrm{G}_{\text {bind }}=\mathrm{G}_{\text {complex }}-\left(\mathrm{G}_{\text {toxin }}+\mathrm{G}_{\text {channel }}\right)
$$

DFIRE [47] software was used for calculating binding affinity. It can be seen from Table 4, that among the three selected scorpion toxins, the HSTX1 showed the maximum binding affinity of $-5.98 \mathrm{Kcal} / \mathrm{mol}$ towards $\mathrm{Kv} 1.3$ channel followed by binding affinity of MTX $-3.67 \mathrm{Kcal} / \mathrm{mol}$ and Pi1 $-3.06 \mathrm{Kcal} / \mathrm{mol}$.

Table 4. Binding Free Energy Calculations between Scorpion Toxins with Kv1.3 Channel

\begin{tabular}{|c|c|}
\hline Scorpion Toxins & Binding Affinity with Kv1.3 Channel (Kcal/mol) \\
\hline \hline HsTX1 & -5.98 \\
\hline MTX & -3.67 \\
\hline Pi1 & -3.06 \\
\hline
\end{tabular}

\section{Disulphide Bridge Pairing Determination and Folding Pattern in Scorpion Toxins}

We obtained two distinct pattern of disulphide bridges arrangement among the selected scorpion toxins. The maurotoxin disulphide bridge pattern is $\mathrm{C} 1-\mathrm{C} 5, \mathrm{C} 2-\mathrm{C} 6, \mathrm{C} 3-$ $\mathrm{C} 7$ and $\mathrm{C} 7-\mathrm{C} 8$ which differs from $\mathrm{C} 1-\mathrm{C} 5, \mathrm{C} 2-\mathrm{C} 6, \mathrm{C} 3-\mathrm{C} 7$ and C4-C8 pattern of Pi1/HsTX1. The residues involved in the disulphide bridges in the MTX are Cys-3-Cys-24, Cys-9Cys-29, Cys-13-Cys-19, Cys-31-Cys-34 whereas a similar residues of HsTX1/Pi1 toxins Cys-3-Cys-24, Cys-9-Cys-29, Cys-13-Cys-31 and Cys-19-Cys-34 are involved. In the selected short-chain four disulphide bridged scorpion toxins acting on Kv1.3 channel, the consensus sequence is different from the three disulphide bridged scorpion toxin by the presence of an extra half-cystine residue within the central part of the sequence which shown as bold in Fig. (4). The remaining half-cystine residue required for the fourth disulphide bridge formation is located at the C-terminal end of the motif. All the three toxins share high sequence identities of $52 \%-68 \%$ between each other. Although these toxins are structurally related to each other, MTX differs in its half-cystine pairings, which are considered as 'nonconventional'. The first two disulphides are C1-C5, C2-C6 are 'conventional' whereas the two others are rearranged to form short cyclic domains one between Cys-13-Cys-19 and another between Cys-31-Cys-34. All the three toxins have eight cycteine residues in conserved positions. The HstX1 and Pil displays the three conserved disulphide bridges found in classical scorpion $\mathrm{K}^{+}$channel inhibitors and the two extra half-cystine residues being cross linked. In the case of MTX only two of the three classical bridges are conserved and the members of the third being paired with each of the two extra half cystine residues.

A mixture of both secondary structures was found in all the three scorpion toxins. Though they differ in their disulphide bridge arrangements, they possess an $\alpha / \beta$ scaffold. In the case of HsTX1 structure the helix is linked to the $\beta$ sheet by the two disulfide bridges Cys-9-Cys-29 and Cys13-Cys-31. The two other disulphide bridges Cys-3-Cys-24 and Cys-19-Cys-34 link the $\mathrm{N}$-terminus to the $\beta$ - sheet and the $\mathrm{C}$-terminus to the loop connecting the $\alpha$ - and $\beta$-structures respectively. In MTX a bended helix from residues Ser-6 to Gln-16 connected by a loop to a two-stranded antiparallel beta sheet residues 23 to 26 and 28 to 31 . In Pil an helical 


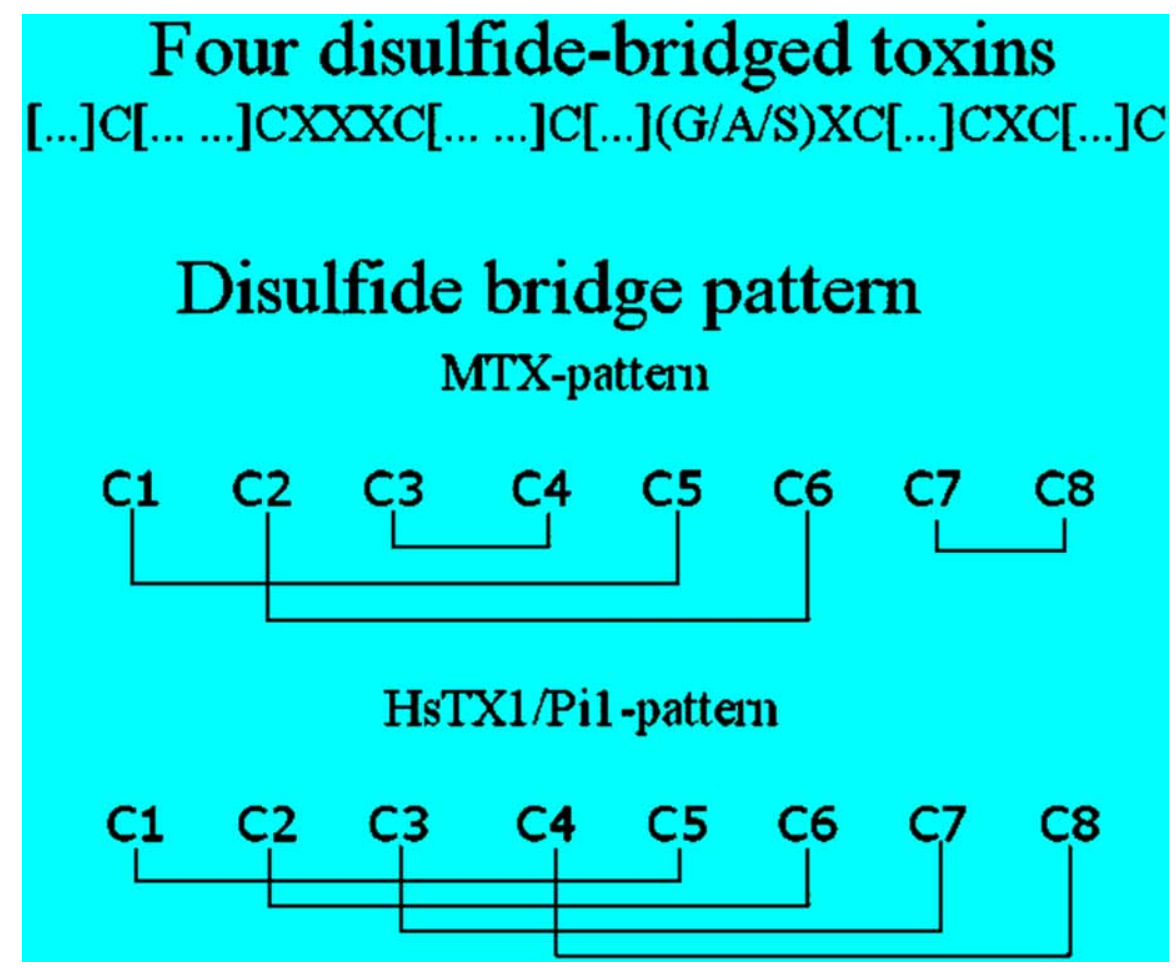

Fig. (4). Consensus motif and disulfide bridge pattern of scorpion toxins.

structure is connected to an antiparallel beta-sheet by two disulfide bridges.

\section{Hydrogen Bond and Hydrophobic Contacts between Scorpion Toxins and Kv1.3 Channel}

During the docking simulations, the hydrogen bonds between scorpion toxins and Kv1.3 channel were analyzed. The average number of hydrogen bonds formed between the toxin and the channel is from 1 to 5 . A list of hydrogen bonds formed between selected scorpion toxins and Kv1.3 channel are shown in Table 5. From this observation the scorpion toxin HsTX1 forming five hydrogen bonds with the Kv1.3 channel. The MTX and Pil form one and three hydrogen bonds with the Kv1.3 channel respectively. The distance between the residues involved in the hydrogen bonds ranges from $2.44 \AA$ to $3.41 \AA$. Similarly the hydrophobic contacts also analyzed. The residues like Ser362, Ser-374, Asp-370, Asp-371, Met-390, His-399, Gly-404 of Kv1.3 channel are involved in hydrophobic contacts with the selected scorpion toxins. The distance between the residues involved in the hydrophobic contacts ranges from $3.36 \AA$ to $3.92 \AA$. The detailed information of hydrophobic contacts are depicted in Table $\mathbf{6}$.

\section{Flexibilities of Scorpion Toxins and Kv1.3 Channel}

To measure the flexibilities of the scorpion toxins and the Kv1.3 channel during docking simulations, we used EINe'mo [52] program. Since the natural structure of the Kv1.3 channel is not so flexible which is embedded in the lipid bilayer, the scorpion toxins are more flexible than the

Table 5. Hydrogen Bond Contacts between Scorpion Toxins and Kv1.3 Channel

\begin{tabular}{|c|c|c|c|c|c|}
\hline \multicolumn{3}{|c|}{ Scorpion Toxins } & \multicolumn{2}{|c|}{ Kv1.3 Channel } & Distance( $(\AA)$ \\
\hline \multirow{3}{*}{ HsTX1 } & Lys-7 & $\mathrm{N}$ & Asp-370 & $\mathrm{O}$ & 3.17 \\
\hline & Arg-27 & $\mathrm{N}$ & Asp-381 & $\mathrm{O}$ & 2.86 \\
\hline & Arg-33 & $\mathrm{N}$ & Asp-398 & $\mathrm{O}$ & 2.76 \\
\hline MTX & Lys-30 & $\mathrm{N}$ & Asp-381 & $\mathrm{O}$ & 2.44 \\
\hline \multirow[t]{2}{*}{ Pi1 } & Lys-3 & $\mathrm{N}$ & Asp-371 & $\mathrm{O}$ & 3.41 \\
\hline & Arg-5 & $\mathrm{N}$ & Asp-371 & $\mathrm{O}$ & 3.37 \\
\hline
\end{tabular}


Table 6. Hydrophobic Contacts between Scorpion Toxins and Kv1.3 Channel

\begin{tabular}{|c|c|c|c|c|c|}
\hline \multicolumn{3}{|c|}{ Scorpion Toxins } & \multicolumn{2}{|c|}{ Kv1.3 Channel } & \multirow{2}{*}{ Distance $(\AA)$} \\
\hline Name & Residue & Atom & Residue & Atom & \\
\hline \multirow[t]{5}{*}{ HsTX1 } & Ser-2 & $\mathrm{C}$ & Ser-362 & $\mathrm{C}$ & 3.84 \\
\hline & Gly-22 & $\mathrm{C}$ & Ser-374 & $\mathrm{C}$ & 3.56 \\
\hline & Met-25 & $\mathrm{C}$ & Asp-370 & $\mathrm{C}$ & 3.24 \\
\hline & Thr-5 & $\mathrm{C}$ & Asp-371 & $\mathrm{C}$ & 3.61 \\
\hline & Lys-28 & $\mathrm{C}$ & Met-390 & $\mathrm{C}$ & 3.93 \\
\hline \multirow[t]{5}{*}{ MTX } & Ser-6 & $\mathrm{C}$ & Ser-362 & $\mathrm{C}$ & 3.84 \\
\hline & Tyr-10 & $\mathrm{C}$ & His-399 & $\mathrm{C}$ & 3.36 \\
\hline & Gly-14 & $\mathrm{C}$ & Ser-362 & $\mathrm{C}$ & 3.81 \\
\hline & Asn-21 & $\mathrm{C}$ & Gly-404 & $\mathrm{C}$ & 3.92 \\
\hline & Tyr-32 & $\mathrm{C}$ & His-399 & $\mathrm{C}$ & 3.74 \\
\hline \multirow[t]{4}{*}{ Pi1 } & Lys-3 & $\mathrm{C}$ & Asp-381 & $\mathrm{C}$ & 3.51 \\
\hline & Asn-22 & $\mathrm{C}$ & Gly-404 & $\mathrm{C}$ & 3.49 \\
\hline & Ile-26 & $\mathrm{C}$ & His-399 & $\mathrm{C}$ & 3.71 \\
\hline & Met-29 & $\mathrm{C}$ & Asp-398 & $\mathrm{C}$ & 3.85 \\
\hline
\end{tabular}

Kv1.3 channel. Therefore the conformational changes of the toxins were considered during the docking simulations. In HsTX1-Kv1.3 complex, the positively charged residues Arg4, Lys-7, Asp-8, Arg-14, Lys-15, Lys-23, Arg-27, Lys-28 and Lys-30 which are involved in the binding shows a maximum stretching range from $0.07 \AA$ to $0.09 \AA$. This is followed by the MTX toxin in which the positively charged residues Thr-4, Lys-7, Arg-14, Lys-15, Lys-23, Lys-27 and Lys-30 involved in binding has stretching range from $0.04 \AA$ to $0.07 \AA$. The residues of Pil toxin Lys-3, Arg-5, Thr-7, Arg-12, Lys-24, Arg-28 and Lys-31 shows the range from $0.02 \AA$ to $0.05 \AA$. The residues of Kv1.3 channel Asp-370, Asp-371, Asp-398 and Asp-381 shows less stretching of 0.02 $\AA$.

\section{DISCUSSIONS}

The main purpose of this study was to determine the complex structures of the four disulphide bridged scorpion toxins with the Kv1.3 potassium channel. Based on the homology model of the human voltage gated potassium channel Kv1.3, we have investigated the recognition between three scorpion toxins and the potassium channel by combining homology modeling and docking simulations. We observed that Ser-362, Asp-370, Asp-371, Ser-374, Asp-381, Met-390, Asp-398, His-399, and Gly-404 are the key residues in Kv1.3 channel for toxins recognitions. In HsTX1 toxin the residues Arg-4, Lys-7, Asp-8, Arg-14, Lys-15, Lys23, Arg-27, Lys-28, Lys-30 are important in blocking the Kv1.3 channel. Similarly in MTX toxin Thr-4, Lys-7, Arg14, Lys-15, Lys-23, Lys-27, Lys-30 and in Pi1 toxin Lys-3, Arg-5, Thr-7, Arg-12, Lys-24, Arg-28, Lys-31 residues are important in the binding towards Kv1.3 channel. The higher in the electrostatic energies between selected scorpion toxins and Kv1.3 channel correspond to the higher in the binding free energy calculations. In addition, we have computed the hydrogen and hydrophobic contacts between the toxins and Kv1.3 channel. Based on the disulphide pattern, folding pattern, electrostatic interaction energies, binding free energies, hydrogen and hydrophobic interactions, we predict that the four-sulphide bridged scorpion toxin HsTX1 has the maximum affinity towards Kv1.3 channel followed by MTX and Pi1. The orientation of HsTX1 toxin has a better blocking ability when compared with other two toxins namely MTX and Pi1. Since our predictions also concede with already published experimental reports, this strategy may be helpful for structural characterization of macromolecular complexes when a high-resolution structure of a complex cannot be obtained by either NMR or X-ray crystallography experiments.

\section{CONCLUSIONS}

The Kv1.3 channel is one of two potassium channels expressed by human $\mathrm{T}$ lymphocytes that are involved in proliferation and cytokine secretion (the other is the calciumactivated $\mathrm{K}+$ channel IKCa1). Researchers at the University of California have recently reported [53] that myelin-reactive encephalitogenic rat $\mathrm{T}$ cells expressed unusually high numbers of Kv1.3 channels following antigenic stimulation. Furthermore adoptive transfer of these $\mathrm{T}$ cells induced multiple sclerosis-like inflammation in rats, an effect which was reduced by Kv1.3 blockade. The search for Kv1.3 blockers or molecules able to prevent channel expression could therefore provide novel anti-inflammatory molecules. Indeed Merck researchers have recently published [54] a series of Kv1.3 channel inhibitors for the potential treatment of autoimmune diseases. Though there are experimental work has been done with rat Kv1.3 channels, we have to go long way for the same work to be done with human ion channels. In this work, the predicted toxin-channel complex 
structures and other computational reports can be used in guiding the future biological studies which involve in design of blocking agents of Kv1.3 channel.

\section{ACKNOWLEDGEMENTS}

The authors gratefully thank the management of Vellore Institute of Technology University for providing the facility to carry out this work. The authors also thank the anonymous reviewers for their valuable comments and suggestions.

\section{ABBREVIATIONS}

$\begin{array}{ll}\text { CHARMM }= & \begin{array}{l}\text { Chemistry at harvard macromolecular } \\ \text { mechanics }\end{array} \\ \text { HsTX1 } & \text { Heterometrus spinnifer toxin } 1 \\ \text { MTX } & =\text { Maurotoxin } \\ \text { Pi1 } & =\text { Pandinus toxin } 1\end{array}$

\section{REFERENCES}

[1] Hille B. Ionic channels of excitable membranes, $2^{\text {nd }}$ ed. Sinauer Associates Inc. Sunderland, MA1992.

[2] Kaczmarek LK, Perney TM. The molecular biology of $\mathrm{K}+$ channels. Curr Opin Cell Biol 1991; 3: 663-70.

[3] Wrisch A, Grissmer S. Structural differences of bacterial and mammalian K+ channels. J Biol Chem 2000; 275: 39345-53.

[4] Biggin PC, Roosild T, Choe S. Pottasium channel structure: domain by domain. Curr Opin Struct Biol 2000; 10: 456-61.

[5] Lu Z, Klem AM, Ramu Y. Ion conduction pore is conserved among potassium channels. Nature 2001; 413: 809-13.

[6] Zhou Y, Morais-cabral JH, Kaufman A, et al. Chemistry of ion coordination and hydration revealed by a $\mathrm{K}+$ channel-Fab complex at $2.0 \mathrm{~A}^{\circ}$ resolution. Nature $2001 ; 414: 43-8$.

[7] Chandy KG, Decoursey TE, Cahalan MD, et al. Voltage-Gated potassium channels are required for human $\mathrm{T}$ lymphocyte activation. J Exp Med 1984; 160: 369-85.

[8] DeCoursey TE, Chandy KG, Gupta S, et al. Voltage-gated K+ channels in human $\mathrm{T}$ lymphocytes: a role in mitogenesis? Nature 1984; 307: 465-8.

[9] Gulbis JM, Zhou M, Mann S, et al. Structure of the cytoplasmic $\beta$ subunit-T1 assembly of voltage-dependent $\mathrm{K}+$ channels. Science 2000; 289: 123-7.

[10] Lewis RS, Cahalan MD. Subset-specific expression of potassium channels in developing murine T lymphocytes. Science 1988; 239: 771-5.

[11] Beeton C, Barbaria J, Giraud P, et al. Selective blocking of voltagegated $\mathrm{K}^{+}$channels improves experimental autoimmune encephalomyelitis and inhibits T cell activation. J Immunol 2001; 166: 93644.

[12] Chandy KG, Cahalan M, Pennington M, et al. Potassium channels in $\mathrm{T}$ lymphocytes: toxins to therapeutic immunosuppresants. Toxicon 2001; 39: 1269-76.

[13] Wickenden AD. $\mathrm{K}^{+}$channels as therapeutic drug targets. Pharmacol Ther 2002; 94: 157-82.

[14] Freudenthaler G, Axmann M, Schindler H, et al. Ultrasensitive pharmacological characterization of the voltage-gated potassium channel Kv1.3 studied by single-molecule fluorescence microscopy. Histochem Cell Biol 2002; 117: 197-202.

[15] Aiyar J, Rizzi JP, Gutman GA, et al. The signature sequence of voltage-gated potassium channels projects into the external vestibule. J Biol Chem 1996; 271: 31013-6.

[16] Doyle DA, Cabral JM, Pfuetzner RA, et al. The structure of the potassium channel: molecular basis of $\mathrm{K}^{+}$conduction and selectivity. Science 1998; 280: 69-77.

[17] MacKinnon R, Reinhart PH, White MM. Charybdotoxin block of shaker $\mathrm{K}^{+}$channels suggests that different types of $\mathrm{K}^{+}$channels share common structural features. Neuron 1988; 1: 997-1001.

[18] Mourre C, Chernova MN, Martin-Eauclaire MF, et al. Distribution in rat brain of binding sites of Kaliotoxin, a blocker of Kv1.1 and Kv1.3 $\alpha$-subunits. J Pharmacol Exp Ther 1999; 291: 943-52.
[19] Peter JM, Varga Z, Hajdu PR, et al. Effects of toxins Pi2 and Pi3 on human T lymphocyte Kv1.3 channels: the role of Glu-7 and Lys24. J Membr Biol 2001; 179: 13-25.

[20] Thompson J, Begenisich T. Electrostatic interaction between charybdotoxin and a tetrameric mutant of shaker $\mathrm{K}^{+}$channels. Biophys J 2000; 78: 2382-91.

[21] Miller C. Neuron. The charybdotoxin family of $\mathrm{K}^{+}$channelblocking. Peptides 1995; 15(1): 5-10.

[22] Romi-Lebrun R, Martin-Eauclaire MF, Escoubas $\mathrm{P}$, et al. Characterization of four toxins from Buthus martensi scorpion venom, which act on apamin-sensitive $\mathrm{Ca}^{2+}$-activated $\mathrm{K}^{+}$channels. Eur J Biochem 1997; 245: 457-64.

[23] Olamendi-Portugal T, Gómez-Lagunas F, Gurrola GB, et al. A novel structural class of $\mathrm{K}^{+}$-channel blocking toxin from the scorpion Pandinus imperator. Biochem J 1996; 315: 977-81.

[24] Kharrat R, Mabrouk K, Crest M, et al. Chemical synthesis and characterization of maurotoxin, a short scorpion toxin with four disulfide bridges that acts on $\mathrm{K}^{+}$channels. Eur J Biochem 1996; 242: 491-8.

[25] Bontems F, Roumestand C, Gilquin B, et al. Refined structure of charybdotoxin: common motifs in scorpion toxins and insect defensins. Science 1991; 254: 1521-3.

[26] Dauplais M, Gilquin B, Possani LD, et al. Determination of the three-dimensional solution structure of noxiustoxin: analysis of structural differences with related short-chain scorpion toxins. Biochemistry 1995; 34: 16563-73.

[27] Alessandri-Haber N, Lecoq A, Gasparini S, et al. Mapping the functional anatomy of $\mathrm{BgK}$ on $\mathrm{Kv} 1.1, \mathrm{Kv} 1.3$, and $\mathrm{Kv} 1.3$. J Biol Chem 1999; 274: 35653-61.

[28] Blanc E, Romi-Lebrun R, Bornet O, et al. Solution structure of two new toxins from the venom of the Chinese scorpion Buthus martensi Karsch blockers of potassium channels. Biochemistry 1998; 37: 12412-8.

[29] Garcia-Calvo M, Leonard RJ, Novick J, et al. Purification, characterization, and biosynthesis of margotoxin, a componenet of centruroides margaritatus venom that selectively inhibits voltagedependent potassium channels. J Biol Chem 1993; 268: 18866-74.

[30] Gilquin B, Racape J, Wrisch A, et al. Structure of the BgK-Kv1.1 complex based on distance restraints identified by double mutant cycles. J Biol Chem 2002; 277: 37406-13.

[31] Goldstein SA, Pheasant DJ, Miller C. The charybdotoxin receptor of a shaker $\mathrm{K}^{+}$channel: peptide and channel residues mediating molecular recognition. Neuron 1994; 12: 1377-88.

[32] Bontems F, Gilquin B, Roumestand C, et al. Analysis of side-chain organization on a refined model of charybdotoxin: structural and functional implications. Biochemistry 1992; 31: 7756-64.

[33] Fernandez I, Romi R, Szendeffy S, et al. Kaliotoxin (1-37) shows structural differences with related potassium channel blockers. Biochemistry 1994; 33: 14256-63.

[34] Krezel AM, Kasibhatla C, Hidalgo P, et al. Solution structure of the potassium channel inhibitor agitoxin 2: caliper for probing channel geometry. Protein Sci 1995; 4: 1478-89.

[35] Harvey AL, Vatanpour H, Rowan EG, et al. Structure-activity studies on scorpion toxins that block potassium channels. Toxicon 1995; 33: 425-36.

[36] Goldstein SA, Colatsky TJ. Ion channels: too complex for rational drug design? Neuron 1996; 12: 1377-88.

[37] Kaczorowski GJ, Garcia ML. Pharmocology of voltage-gated and calcium-activated potassium channels. Curr Opin Chem Biol 1999; 3: 448-58.

[38] Tytgat J, Chandy KG, Garcia ML, et al. A unified nomenclature for short-chain peptides isolated from scorpion venoms: alpha-KTx molecular subfamilies. Trends Pharmacol Sci 1999; 20(11): 444-7.

[39] Savarin P, Romi-Lebrun R, Zinn-Justin S, et al. Structural and functional consequences of the presence of a fourth disulfide bridge in the scorpion short toxins: solution structure of the potassium channel inhibitor HsTX1. Protein Sci 1999; 8: 2672-85.

[40] Blanc E, Sabatier JM, Kharrat R, et al. Solution structure of maurotoxin, a scorpion toxin from Scorpio maurus, with high affinity for voltage-gated potassium channels. Proteins 1997; 29: 321-3.

[41] Carrega L, Mosbah A, Ferrat G, et al. The impact of the fourth disulfide bridge in scorpion toxins of the alpha-KTx6 subfamily. Proteins 2005; 61: 1010-23.

[42] Berman HM, Westbrook J, Feng Z, et al. The Protein data bank. Nucleic Acids Res 2000; 28: 235-42. 
[43] Thompson JD, Higgins DG, Gibson TJ. CLUSTAL W: improving the sensitivity of progressive multiple sequence alignment through sequence weighting, position-specific gap penalties and weight matrix choice. Nucleic Acids Res 1994; 22: 4673-80.

[44] Tovchigrechko A, Vakser IA. Development and testing of an automated approach to protein docking. Proteins 2005; 60(2): 296301.

[45] Bernard Brooks R, Robert Bruccoleri E, Barry Olafson D, et al. CHARMM: A program for macromolecular energy, minimization and dynamics calculations. J Comput Chem 1983; 4(2): 87-217.

[46] Schafer H, Van Gunsteren WF, Mark AE. Estimating relative free energies from a single ensemble: hydration free energies. J Comput Chem 1999; 20: 1604-17.

[47] Liu S, Zhang C, Zhou H, et al. A physical reference state unifies the structure-derived potential of mean force for protein folding and binding. Protein 2004; 56: 93-101.

[48] Ferre F, Clote P. Disulfide connectivity prediction using secondary structure information and diresidue frequencies. Bioinformatics 2005; 21: 2336-46.
[49] Ferre F, Clote P. DiANNA: a web server for disulfide connectivity prediction. Nucleic Acids Res 2005; 33: W230-W232.

[50] Hong-Bin Shen, Kuo-Chen Chou. Ensemble classifier for protein folding pattern recognition. Bioinformatics 2006; 22: 1717-22.

[51] Alexander Diemand A, Holger Scheib. iMolTalk: an interactive, internet-based protein structure analysis server. Nucleic Acids Res 2004; 32: 512-6.

[52] Karsten S, Yves-Hendri S. ElNemo: a normal mode web server for protein movement analysis and the generation of templates for molecular replacement. Nucleic Acids Res 2004; 32: W610-4.

[53] Beeton C, Heike W, Singh S, et al. A Novel Fluorescent Toxin to Detect and Investigate Kv1.3 Channel Up-regulation in chronically activated T lymphocytes. J Biol Chem 2003; 278: 9928-37.

[54] Bao J, Miao S, Kayser F, et al. Potent Kv1.3 inhibitors from correolide-modification of the C18 position. Bioorg Med Chem Lett $2005 ; 17: 447-51$. 